\title{
Neonatal spleen rupture, shock, and factor vii deficiency
}

\begin{abstract}
Background: Severe symptomatic factor VII deficiency is very rare. Spleen rupture in a neonate with factor VII deficiency is even rarer. Only one case of hemoperitoneum and intracranial hemorrhage in a neonate with this disease is reported in the literature. Furthermore, the manifestation of hemoperitoneum by abdominal distention, abdominal tenderness, and anemia is often delayed after splenic rupture, and is seldom recognized before the onset of hypovolemic shock.
\end{abstract}

Case: We present the case of a term 24-hour-old male newborn admitted to our neonatal intensive care unit for pallor and vital distress. He was diagnosed with perinatal splenic rupture, and underwent splenectomy owing to hemodynamic instability. A cephalohematoma was also noted, after a traumatic delivery with serial compressions on the mother's abdomen during labor. In addition, coagulation abnormalities were noticed on repeated testing, with consistently prolonged prothrombin time and normal partial thromboplastin time. Subsequent workup diagnosed congenital factor VII deficiency, with a $2 \%$ activity level in the plasma, and a heterozygous missense mutation (exon 8 of the gene c.1109G $>\mathrm{T}$ p.Cys370Phe; previously reported as $\mathrm{C} 310 \mathrm{~F})$.

Conclusion: Factor VII deficiency is a rare condition with variable clinical presentations, usually poorly correlated to the factor's activity levels in the plasma. However, most severe bleedings usually occur early in infancy. Our term newborn presented with two sites of bleeding: the cephalohematoma, and the hemoperitoneum from his ruptured spleen. It is not clear whether those two bleeding incidents were related to his factor VII deficiency; or if they were independent discoveries, simply indicating a traumatic delivery.

Keywords: neonate, spleen rupture, hemoperitoneum, congenital factor vii deficiency.
Volume 6 Issue 6 - 2017

\author{
Abou Zeid C,' Abou Zeid M, ${ }^{2}$ Loutfi G, ${ }^{3}$ \\ Yaacoub $A,{ }^{4}$ Noun $P^{5}$ \\ 'Saint Joseph University Faculty of Medicine, Hôtel-Dieu de \\ France Hospital, Lebanon \\ ${ }^{2}$ Chief of the Neonatal Intensive Care Unit, Lebanese American \\ University Medical Center Rizk Hospital, Lebanon \\ ${ }^{3}$ Department of Pediatric Surgery, Al-Hayat Hospital, Lebanon \\ ${ }^{4}$ Department of Radiology, Al-Hayat Hospital, Lebanon \\ ${ }^{5}$ Department of Pediatric Hematology and Oncology, Saint \\ George Hospital University Medical Center, Lebanon
}

Correspondence: Abou Zeid C, Saint Joseph University Faculty of Medicine, Hôtel-Dieu de France Hospital, Achrafieh, Lebanon, Email peternoun@gmail.com

Received: May 17, 2017 | Published: May 23, 2017

\section{Introduction}

While splenic rupture in a newborn is infrequent, its consequences can be lethal. Clinicians should have a high index of suspicion, especially in case of a history of possible trauma before or during delivery, even with a normal spleen. Common causes of spleen rupture in a neonate are birth trauma from difficult or even normal vaginal delivery, premature or large birth weight infants, erythroblastosis fetalis, wandering spleen syndrome, maternal trauma, maternal anticonvulsivant therapy, and some idiopathic cases. Abdominal imaging such as ultrasound or CT scan can further confirm the diagnosis. Conservative management is preferred when possible, to avoid the infectious risk associated with total splenectomy. We present here the case of a newborn, admitted to neonatal intensive care unit (NICU), at 24hours of life for neonatal shock.

\section{Case report}

The mother (G2P2A0) was 26years old, with an A positive blood group. She had given birth to her first girl at term (40weeks of gestation) by spontaneous vaginal delivery. The girl had a birth weight of $4,300 \mathrm{~g}$ without any incidents in her medical history. The boy discussed in our case was born at term (39weeks of gestation and 4days), with cephalic presentation, and difficult extraction. His birth weight was 4,200g. His Apgar score was 9 at the first minute, and 10 at 5 minutes of life, with an immediate cry after birth. No pediatrician was present during delivery. He was then transferred to the nursery. At 24hours of life, when admitted to the NICU, the baby presented with tachycardia (heart rate: $180 / \mathrm{min}$ ), tachypnea (respiratory rate: $60 / \mathrm{min}$ ), and a temperature of $37.9^{\circ} \mathrm{C}$ with an oxygen saturation of $92 \%$ under
$2 \mathrm{~L}$ of oxygen by nasal cannula. He had a capillary refill time of more than 6 seconds, and his blood pressure measurement was impossible to be performed. He was pale, with discoloration of his skin, grunting, nasal flaring and respiratory distress (Silverman's score: 5). He also presented with an extensive parietal cephalohematoma. Resuscitation was immediately started with oxygen by nasal cannula, catheterization of the umbilical vein, perfusion of D10 with calcium, and a regimen of three intravenous antibiotics: ampicillin $150 \mathrm{mg} / \mathrm{kg} / \mathrm{day}$, cefotaxime $150 \mathrm{mg} / \mathrm{kg} /$ day, and amikacin $15 \mathrm{mg} / \mathrm{kg} /$ day. His blood tests, drawn urgently, showed an hemoglobin $(\mathrm{Hb})$ count of $4 \mathrm{~g} / \mathrm{dL}$, hematocrit: $14 \%$, with a mean corpuscular volume of $101 \mathrm{fl}$. His white blood cells were $52,500 / \mathrm{mm} 3$, platelets: $221,000 / \mathrm{mm} 3$. His prothrombin time (PT) was prolonged: $36.7 / 11.5$; INR at 4.5 ; with a normal partial thromboplastin time (PTT) of 24/22. His arterial blood gas showed: pH: 6.97, pCO2: $18 \mathrm{~mm} \mathrm{Hg}$, pO2: $85 \mathrm{~mm} \mathrm{Hg}, \mathrm{Hb}: 3.6 \mathrm{~g} / \mathrm{dL}, \mathrm{HCO}^{-}: 4$ $\mathrm{mmol} / \mathrm{L}$, base excess: $-25 \mathrm{mmol} / \mathrm{L}$. He had a severe metabolic acidosis with anemia. He received two consecutive transfusions of $\mathrm{O}$ negative packed red blood cells $(20 \mathrm{~mL} / \mathrm{kg} /$ transfusion $)$, with minimal increase of his hemoglobin to $5.4 \mathrm{~g} / \mathrm{dL}$.

At hour 10 after his admission to the NICU, he showed acute abdominal enlargement along with abdominal tenderness. He became oliguric (diuresis: $0.3 \mathrm{~mL} / \mathrm{kg} / \mathrm{h}$ ) and an intra-abdominal hemorrhage was suspected. An abdominal ultrasound was performed and showed evidence of abundant free peritoneal fluid with laceration and rupture of his spleen (Figure 1). A transfontanellar ultrasound was also performed, and showed no intraventricular hemorrhage. Transfusions were continued with no improvement of his anemia. The baby was rushed to surgery considering his hemodynamic instability, and 
abundant hemoperitoneum. A spleen fracture was noted. The surgeon performed a total splenectomy with ligation of the vessels under general anesthesia to control the bleeding, with no possibility of conservative treatment. The neonate had a favorable post-operative evolution with return to stable hemodynamic status after surgery, and his anemia was corrected. The amikacin was stopped at day3, and the other two antibiotics were continued until day12. He stayed in the NICU for 17 days, where laboratory testing at days 3, 5, 8 and 13 of life, still showed prolonged PT and INR, with normal PTT. A pediatric hematologist was consulted at day15. Fibrinogen levels as well as D-dimers were within normal limits.

The workup eventually showed a congenital deficiency in coagulation factor VII. The factor VII activity level in the plasma was $2 \%$. The preliminary molecular results showed a mutation in factor VII: presence of a missense mutation in heterozygous state in the exon 8 of the gene c.1109G $>$ T p.Cys370Phe (previously reported as C310F). There was no identifiable family history of coagulopathy. As the quality of DNA samples was very poor, the concentration was low and the absorbance at $230 \mathrm{~nm}$ showed a strong contamination of phenols. Therefore, additional DNA analysis is being performed as of 30 April 2017 in Italy to check the DNA not only with the agarose gel, but also using a spectrophotometric measurement at $260 \mathrm{~nm}$ and $280 \mathrm{~nm}$ to measure the protein contamination, and at $230 \mathrm{~nm}$ to measure the phenols contamination. Data shall be available within 3 months. Retrospective interrogatory revealed that the labor had stopped at full cervical dilatation, and that the mother's abdomen was compressed numerous times to expedite the baby's delivery, probably causing traumatism to the newborn's spleen. The splenectomized patient was then given anti pneumococcal, anti-meningococcal, and anti-haemophilus influenzae B vaccination. After a follow-up of six years, the only notable incident related to his congenital factor VII deficiency was a single episode of self-limiting mild epistaxis. The patient needed factor replacement during his first year only, and never after that.

\section{Discussion}

Splenic rupture in the neonatal period is a rare occurrence, and is seldom reported in the literature..$^{1,2}$ Although some cases can be spontaneous, the most common etiology encountered is traumatic. ${ }^{2}$ Antenatal or birth traumatism during a difficult delivery can cause rupture of the spleen even to a normal spleen without splenomegaly or other underlying disease. ${ }^{3}$ The excessive pressure in the thorax of the baby during a traumatic delivery can force the spleen from under the rib cage into the abdominal cavity, causing tension to its supporting ligaments. ${ }^{4}$ The rupture of the spleen is thought to occur in two stages: after a compression or a traumatic injury, a sub capsular hematoma forms. It can then progress within hours or days to a rupture of the spleen capsule, leading to hemorrhagic shock. ${ }^{4,5}$ This two-step mechanism can explain the delayed onset of the classic triad: anemia, abdominal distention, and abdominal tenderness in patients with splenic rupture. ${ }^{6}$ In such circumstances, abdominal imaging such as ultrasound or CT scan if the patient is stable should be promptly performed to ensure rapid intervention and treatment. Conservative management of splenic rupture is the preferred approach when possible, in order to avoid the immunocompromised state associated with splenectomy in newborns. ${ }^{7}$ However, in our case, considering hemodynamic instability and continuous bleeding, splenectomy had to be performed.

We believe that in our case, rupture of the spleen was a consequence of external traumatism, considering the duration of fetal dystocia and the extrinsic compressions on the mother's abdomen. The fact that an equal birth term sister with higher weight was born by spontaneous vaginal delivery with no incidents corroborates further our hypothesis. We believe that if confronted to an impacted fetal head after a prolonged second stage of labor with ruptured membranes, a cesarian section should be performed with abdomino-vaginal delivery. ${ }^{8}$ The fetus' head should be lifted using the gynecologist's cupped hand into a transverse uterine incision to allow for extraction without the risk of traumatism by forceps or other maneuvers. Extrinsic compressions on a mother's abdomen during obstructed labor should be prohibited.

Factor VII deficiency is a rare autosomal recessive coagulation disorder, ${ }^{9}$ and its frequency is higher in countries where consanguineous marriage is more common, ${ }^{10}$ such as in Lebanon. However, the parents in our case were not consanguineous, and no family history of bleeding disorder could be identified. This coagulation factor deficiency's presentation can vary from totally asymptomatic patients to patients having severe life-threatening bleeding. ${ }^{11}$ Frequent presentations are prolonged bleeding from epistaxis, easy bruising, gum bleeding, muscle hematoma, gastro-intestinal bleeding, and bleeding in the central nervous system; the last two being the most common manifestations in infancy. ${ }^{9}$ Severe bleeding from factor VII deficiency usually occurs early in life, while moderate to mild phenotypes presents later in life. ${ }^{12}$ Patients with major bleeds have a higher risk of life-long bleeding events. ${ }^{13}$ Also, a poor correlation between clinical symptoms and factor VII activity levels in the plasma is described, ${ }^{14}$ and less than 5 cases of neonatal spleen rupture associated with coagulation disorders are reported in the literature. These cases discuss patients with factor VIII deficiency, also known as hemophilia A. ${ }^{15,16}$ Only one case reporting hemoperitoneum and intracranial hemorrhage as complications of factor VII deficiency is reported in a newborn ${ }^{17}$. In our patient's case, the coagulation studies were repeatedly abnormal with an elevated PT and INR, and low activated PTT. This discordance evokes the diagnosis of factor VII deficiency, which is confirmed by dosage of the factor. ${ }^{9}$ Our patient presented two sites of bleeding: the cephalohematoma, and the hemoperitoneum from the ruptured spleen. It is not clear whether or not those two bleeding incidents were related to his factor VII deficiency; or if they were independent discoveries with no correlation to his coagulation disorder, simply indicating a traumatic delivery.

\section{Acknowledgments}

None.

\section{Conflicts of interest}

Author declares there are no conflicts of interest.

\section{Funding}

None.

\section{References}

1. Tengsupakul S, Sedrak A, Freed J, et al. Splenic rupture in a newborn with severe hemophilia--case report and review. J Pediatr Hematol Oncol . 2010;32(4):323-326.

2. Bickler S, Ramachandran V, Gittes GK, et al. Nonoperative management of newborn splenic injury: a case report. J Pediatr Surg. 2000;35(3):500-501.

3. Gruenwald P. Rupture of liver and spleen in the newborn infant. $J$ Pediatr. 1948;33(2):195-201.

4. Chryss C, Aaron WS. Successful treatment of rupture of normal spleen in newborn. Am J Dis Child. 1980;134(4):418-419. 
5. Hui CM, Tsui KY. Splenic rupture in a newborn. J Pediatr Surg 2002;37(4)

6. Delta BG, Eisenstein EM, Rothenberg AM. Rupture of a normal spleen in the newborn: report of a survival and review of the literature. Clin Pediatr (Phila). 1968;7(6):373-376.

7. Bairdain S, Litman HJ, Troy M, et al. Twenty-years of splenic preservation at a level 1 pediatric trauma center. $J$ Pediatr Surg . 2015;50(5): 864-868.

8. Landesman R, Graber EA. Abdominovaginal delivery: Modification of the cesarean section operation to facilitate delivery of the impacted head. Am Obstet Gynecol . 1984;148(6):707-710.

9. Mariani G, Bernardi F. Factor VII Deficiency. Semin Thromb Hemost . 2009;35(4):400-406.

10. Mannucci PM, Duga S, Peyvandi F. Recessively inherited coagulation disorders. Blood. 2004;104(5):1243-1252.

11. Mariani G, Napolitano M, Dolce A, et al. Replacement therapy for bleeding episodes in factor VII deficiency. A prospective evaluation. Thromb Haemost . 2013;109(2):238-247.
12. Mariani G, Herrmann FH, Dolce A, et al. Clinical phenotypes and factor VII genotype in congenital factor VII deficiency. Thromb Haemost. 2005;93(3):481-487.

13. Di Minno MND, Dolce A, Mariani G, et al. Bleeding symptoms at disease presentation and prediction of ensuing bleeding in inherited FVII deficiency. Thromb Haemost. 2013;109(6):1051-1059.

14. Hunault M, Bauer KA. Recombinant factor VIIa for the treatment of congenital factor VII deficiency. Semin Thromb Hemost . 2000;26(4):401-405.

15. Adamu I, Xiao P, Asarian A. Splenic rupture and intracranial haemorrhage in a haemophilic neonate: Case report and literature review. Afr J Paediatr Surg. 2012;9(2):163-165.

16. Johnson-Robbins LA, Porter JC, Horgan MJ. Splenic rupture in a newborn with hemophilia A: case report and review of the literature. Clin Pediatr (Phila) . 1999;38(2):117-119.

17. Horng YC, Chou YH, Chen RL, et al. Congenital factor VII deficiency complicated with hemoperitoneum and intracranial hemorrhage: report of a case. J Formos Med Assoc Taiwan Yi Zhi . 1993;92(1):85-87. 